nephron

Practice
Nephron 2015;129:219-222

DOI: $10.1159 / 000381196$
Received: January 28, 2015

Accepted after revision: February 9, 2015

Published online: March 10, 2015

\title{
Is 'Bad Luck' an Important Determinant of Cancer Incidence and Does This Concept Apply to Kidney Tumors?
}

\author{
Enrico Garattini ${ }^{a} \quad$ Alessandra Tavani $^{\text {b }}$ \\ a Laboratory of Molecular Biology, Department of Biochemistry and Molecular Pharmacology and bepartment of \\ Epidemiology, IRCCS-Istituto di Ricerche Farmacologiche 'Mario Negri', Milano, Italy
}

\begin{abstract}
Key Words
Cancer · Tumor incidence $\cdot$ Kidney cancer $\cdot$ Stem cell . Replication
\end{abstract}

\begin{abstract}
A study recently published in Science demonstrates a strong correlation between the total number of stem cell divisions and the lifetime risk of cancer in various organs. The tumors considered are divided into two classes, that is, D (Deterministic) and R (Replicative). Stochastic factors presumably attributable to errors deriving from DNA replication are proposed to be at the basis of R-tumor frequency, leading to the conclusion that 'bad luck' is a primary determinant of certain types of cancer. The present Second Opinion highlights potential problems associated with the hypothesis of the study, as some of these also apply to kidney cancer. The aim is to point out that chance is not a major cause of cancer incidence because it is not substantiated by the data available through epidemiological evidence. In particular, we highlight that differences in tumor incidence associated with sex and geographic areas are not in line with the 'bad luck' hypothesis. Further aspects of tumor biology that do not entirely fit with the idea that stochastic events related to DNA replication of normal stem cells are the heterogeneity of the cancer cell phenotype and the heterogeneity of intra-organ localization, often observed in tumors originating from the
\end{abstract}

same tissue. From a public health perspective, the points discussed here as well as the absence of data on prevalent tumors, like breast and prostate cancer, do not support the oversimplified message conveyed by the media that the majority of cancer cases cannot be prevented.

(C) 2015 S. Karger AG, Basel

In a provocative study published in Science very recently [1], Tomasetti and Vogelstein report the existence of a strong correlation between the calculated total number of stem cell divisions in a given organ and the lifetime risk of cancer in the same organ. The work is based on the analysis of 31 tumors arising in different tissues for which the number of stem cell divisions can be easily and reliably deduced from the available scientific literature. Although the analysis includes two big killers like lung cancer, which is analyzed in both the smoking and nonsmoking population, and colorectal cancer, it does not include the two other most frequent tumors, that is, breast and prostate cancer. Of particular relevance for the present commentary, the study does not contain any data on kidney cancer. The different tumors considered are divided by the authors into two classes, D (Deterministic, 9 types of tumors) and R (Replicative, 22 types of tumors). Deterministic factors, such as environmental mutagens and hereditary predisposition are proposed to play a ma-

\section{KARGER 125}

(c) 2015 S. Karger AG, Base

$1660-8151 / 15 / 1293-0219 \$ 39.50 / 0$

E-Mail karger@karger.com

www.karger.com/nef
Dr. Enrico Garattin

Laboratory of Molecular Biology, Department of Biochemistry and Molecular Pharmacology IRCCS-Istituto di Ricerche Farmacologiche 'Mario Negri' via La Masa 19, IT-20156 Milano (Italy)

E-Mail enrico.garattini@ marionegri.it 
jor role in determining the risk of D-tumors. In contrast, stochastic factors presumably attributable to errors deriving from DNA replication are implicated as primary determinants of R-tumor incidence. Given the fact that the majority of the tumors analyzed fall within the R group, the authors propose that random mutations generated during the process of DNA replication in normal and non-cancerous stem cells are responsible for two thirds of the tumors considered. On the basis of the data collected, the authors propose that 'bad luck' may be a significant factor in the incidence of certain types of tumors. The study provides a new hypothesis as to the basic mechanisms underlying the genesis of various types of cancers. Predominantly because of the serious ramifications within the context of cancer prevention, the article and the corresponding editorial [2] have been extensively covered by media and have spurred mixed reactions by the scientific community [3]. The present Second Opinion aims at highlighting some of the potential confounding factors associated with the analysis performed in the study, as these may apply to kidney cancer as well. In particular, it is in our scope to point out that the oversimplified message conveyed by the media that the 'the majority of cancer is due to bad luck, hence there is nothing we can do about it' is not supported by the data contained in the Science article and by the overall epidemiological evidence. Thus, the hypothesis should not be used to diminish the significance of the current efforts in primary cancer prevention.

In the article by Tomasetti and Vogelstein, sex differences are not taken into consideration in the analysis performed and this parameter may weaken the hypothesis that stochastic factors are major determinants in the appearance of tumors belonging to the R group. Except for ovarian and testicular cancer, all the tumors that are used to correlate the total stem cell divisions and lifetime risk do not involve genital tissues and organs. Nevertheless, the incidence of some of the tumors arising from nongenital tissues representing the object of the study is different in males and females. For instance, the incidence of the R-type esophageal cancer in the United States is approximately 5.5/100,000 males, while a frequency of $1.1 / 100,000$ is calculated for females. Similar differences in the sex-specific incidence of esophageal cancer are confirmed in all the other continents, as indicated by the data available for the United Kingdom, Italy, South Africa and Australia (http://globocan.iarc.fr/ia/World/atlas.html). Although data on possible sex-related differences in the number and proliferation potential of esophageal stem cells are not available, it is highly unlikely that males and females are characterized by five-fold differences in this parameter. Remarkable sex-specific variations in the incidence of two other R-tumors, like liver and thyroid cancers, are also well known (http://globocan.iarc.fr/ia/ World/atlas.html). Interestingly, a similar situation applies to kidney cancer although sex-related differences in this tumor are less marked than in the aforementioned types of neoplasia. In fact, a twofold difference in the incidence of kidney cancer is observed throughout the world, as males are significantly more affected by this tumor than females (new cases/100,000 individuals in US: males $=15.9$; females $=8.5$ ). At present, the reasons for the sex-dependent differences in the R-type liver, thyroid and esophageal tumors as well as kidney cancer are largely unknown although they may be related to hormonal factors, lifestyle and nutrition habits, which can be modified and represent the target of primary prevention schemes. Indeed, as far as esophageal cancer is concerned, tumor incidence is highly correlated with sex-associated life-style characteristics, such as smoking habits and alcohol drinking, which are easily modifiable factors [4]. On the basis of what is discussed in this section, sex-related differences in the incidence of kidney cancer point to the fact that tumors arising in this organ are unlikely to belong to the R-group.

Another important aspect of tumor epidemiology that is not taken into account by the study and may not be entirely in line with the hypothesis that incidence of Rgroup tumors is primarily due to chance is the geographical variability of this epidemiologic index. The incidence (per 100,000 individuals) of the R-type esophageal cancer in the male population is more than threefold higher in China (18.6) than in the United States (5.5) and Australia (5.4). A high frequency of this tumor is also observed in the southeastern region of Africa, being extremely high in Kenya (20.5). In addition, the incidence of thyroid cancer in the United States, Canada and the United Kingdom is worth noticing, as values of 6.5, 4.8 and 1.5 , respectively, are observed in the three countries. This geographical variability hints to genetic predisposition or environmental factors playing a more important role than simple chance in the lifetime risk of esophageal and thyroid cancers. By the same token, it is difficult to reconcile the vast difference in the incidence of the $\mathrm{R}$ type hepatocellular carcinoma between the US (9.8), Norwegian (2.9) and Swedish (3.4) male populations, with differences in the number and proliferation rate of liver stem cells. Thus, geographical and ethnic differences are other parameters that do not necessarily fit with the idea that chance and stochastic factors associated 
with stem cell DNA replication are major determinants of R-type tumors. As for kidney cancer, the incidence of this tumor is also subject to geographical differences. For instance, US males (15.9) have an approximately fourfold higher risk than the Brazilian (3.9) counterpart to suffer from kidney cancer. These figures support the importance of the environmental and genetic factors proposed to be associated with an increased risk of kidney cancer such as smoking, regular use of NSAIDs, obesity, faulty genes, a family history of kidney cancer, kidney stones and high blood pressure [5-7]. Taken together, these factors explain about half of all renal cell cancer cases $[8,9]$, while the remainder may be due to unknown factors other than chance. In any case, geographical differences in the incidence represent a second parameter suggesting that kidney cancer is more likely to belong to the $\mathrm{D}$ - than to the R-type of tumors.

A further aspect of tumor biology that is not completely consistent with the concept that stochastic events related to DNA replication of cancer stem cells is the heterogeneity of the tumor cell phenotype often observed in tumors originating in the same tissue and presumably from the same stem cell compartment. For instance, both luminal and triple-negative breast cancers should initially derive from the same pluripotent stem cell [10]. Thus, we would expect the same frequency of luminal and triple-negative cancer, if stochastic events linked to DNA replication of normal stem cells were at the basis of both tumor subtypes. Clearly, this is not the case and luminal tumors are 6-7 fold more frequent than the triple negative counterparts. As breast cancer is not included in the study, it is worth mentioning here that glioblastoma, which is classified as an R-type tumor is likely to represent another example of the discussed phenotypic heterogeneity. In fact, at least 10 different subtypes of glioblastoma are known, such as giant cell glioblastoma or pilocytic astrocytoma, and they have different incidences. It would be interesting to know whether the same or different stem cells are basically responsible for the formation the two most common kidney tumors, that is, renal cell carcinoma, which represents over $80 \%$ of the neoplastic diseases arising in this organ, and transitional cell carcinoma, which accounts for the majority of the remainder (http:// en.wikipedia.org/wiki/Kidney_cancer). Another example of heterogeneity that challenges the stochastic theory of cancer incidence relates to the intra-organ localization of the tumor. The point is exemplified by gastric cancer, which is not included in the study, and, once again, glioblastoma. For instance, the vast majority of stomach tumors localize to the lesser curvature and only a minority is found in the larger curvature. By the same token, glioblastoma is most often localized to the subcortical white matter of the cerebral hemispheres. Intra-ventricular [11], cerebellar, spinal glioblastomas [12] and brainstem glioblastomas [13] are infrequent. It is worth mentioning that differences in intra-organ localization of kidney cancers are known, which is again in line with the idea that these tumors are likely to be classified in the $\mathrm{D}$-group.

In the interest of space, we discussed only some of the major points that do not fit with the theory proposed by Tomasetti and Vogelstein, although other general and specific aspects of the study would deserve further discussion. Nevertheless, on the basis of the few points raised, we propose that stem cell divisions and chance are unlikely to be major factors in the genesis of kidney cancer. However, the issue is still open and calls for specific studies, as recent epidemiologic studies indicate that chronic kidney disease, which is purported to be associated with chronic activation of kidney stem cell proliferation, is an important risk factor for renal cancer [14], with particular reference to papillary renal carcinoma [15]. With respect to this last type of tumor, it is interesting to note that it was recently proposed that papillary renal carcinoma may originate from renal stem cells [16]. At present, one of the main problems in this area is represented by the fact that we still lack basic knowledge on the number, the characteristics and the intra-organ localization of normal kidney stem cells [16]. No matter what is the real role of stem cell division in the genesis of kidney cancer, the theory should not hamper or slowdown studies aimed at determining major and preventable risk factors for this tumor.

We would like to conclude with a general consideration regarding the coverage of the study provided by mass-media, since it represents an example of bad scientific communication. In fact, the oversimplified message conveyed to the layman that the majority of cancer cases cannot be prevented, a statement which is surprisingly present also in the Science Editorial of the Tomasetti and Vogelstein article [2], cannot be deduced from the original Science article. Indeed, the authors demonstrate that the proportion of cancer risk variation in different tissues can be partially explained by the rate of division of the stem cells in that tissue. Obviously, this does not mean that cancer depends only on 'bad luck', as claimed by the media. In simple terms, it is true that a smoker has a much higher risk than a non-smoker to contract lung cancer. Nevertheless and consistent with a larger lifetime number of stem cell divisions, lung cancer is much more frequent than bone or thyroid cancer even in non-smokers. There is another point that should be considered, as it was en- 
tirely distorted in the coverage provided by media. Even if chance were indeed an important determinant of the tumor types analyzed by Tomasetti and Vogelstein, which is yet to be unequivocally demonstrated, the concept seems to apply only to relatively infrequent cancer types and certainly not to the majority of oncologic patients, as claimed by the media and by some authoritative commentators. In fact, as already remarked, two of the most frequent tumors, breast and prostate cancer, are not con- sidered by the study. In addition, smoking and certainly not chance is the principal cause of lung cancer, the third most frequent tumor, as discussed earlier and acknowledged by these same authors in the Science article. Thus, from a public health perspective, the claim that the majority of cancer patients should place the blame on 'bad luck' is not only wrong, but it is also dangerous because it is likely to hamper efforts in programs aimed at primary tumor prevention.

\section{References}

1 Tomasetti C, Vogelstein B: Cancer etiology. Variation in cancer risk among tissues can be explained by the number of stem cell divisions. Science 2015;347:78-81.

-2 Couzin-Frankel J: Biomedicine. The bad luck of cancer. Science 2015;347:12.

>3 Couzin-Frankel J: Science communication. Backlash greets 'bad luck' cancer study and coverage. Science 2015;347:224.

4 Engel LS, Chow WH, Vaughan TL, Gammon MD, Risch HA, Stanford JL, Schoenberg JB, Mayne ST, Dubrow R, Rotterdam H, West AB, Blaser M, Blot WJ, Gail MH, Fraumeni JF Jr: Population attributable risks of esophageal and gastric cancers. J Natl Cancer Inst 2003; 95:1404-1413.

5 Lipworth L, Tarone RE, McLaughlin JK: The epidemiology of renal cell carcinoma. J Urol 2006;176:2353-2358.
6 McLaughlin JK, Lipworth L, Tarone RE: Epidemiologic aspects of renal cell carcinoma. Semin Oncol 2006;33:527-533.

7 Cheungpasitporn W, Thongprayoon C, O'Corragain OA, Edmonds PJ, Ungprasert P, Kittanamongkolchai W, Erickson SB: The risk of kidney cancer in patients with kidney stones: a systematic review and meta-analysis. QJM 2015;108:205-212.

$>8$ Benichou J, Chow WH, McLaughlin JK, Mandel JS, Fraumeni JF Jr: Population attributable risk of renal cell cancer in Minnesota. Am J Epidemiol 1998;148:424-430.

-9 Tavani A, Negri E, La Vecchia C: Re: 'Population attributable risk of renal cell cancer in Minnesota'. Am J Epidemiol 1999;150:222.

10 Polyak K: Heterogeneity in breast cancer. J Clin Invest 2011;121:3786-3788.

11 Lee TT, Manzano GR: Third ventricular glioblastoma multiforme: case report. Neurosurg Rev 1997;20:291-294.
12 Kleihues P, Cavenee WK (eds): Pathology and genetics of tumours of the nervous system, world health organization classification of tumours. Lyon, France, IARC Press, 2000.

13 Dohrmann GJ, Farwell JR, Flannery JT: Glioblastoma multiforme in children. J Neurosurg 1976;44:442-448.

14 Lowrance WT, Ordonez J, Udaltsova N, Russo $\mathrm{P}, \mathrm{Go}$ AS: CKD and the risk of incident cancer. J Am Soc Nephrol 2014;25:2327-2334.

$>15$ Woldu SL, Weinberg AC, RoyChoudhury A, Chase H, Kalloo SD, McKiernan JM, DeCastro GJ: Renal insufficiency is associated with an increased risk of papillary renal cell carcinoma histology. Int Urol Nephrol 2014;46: 2127-2132.

16 Axelson H, Johansson ME: Renal stem cells and their implications for kidney cancer. Semin Cancer Biol 2013;23:56-61. 